\title{
Delivery of sodium morrhuate to hemangioma endothelial cells using immunoliposomes conjugated with anti-VEGFR2/KDR antibody
}

This article was published in the following Dove Press journal:

International Journal of Nanomedicine

19 September 2017

Number of times this article has been viewed

\author{
Xiaoli Li' \\ Xiaoyong Ren ${ }^{2}$ \\ Jianmin Liang ${ }^{2}$ \\ Weijun $\mathrm{Ma}^{2}$ \\ Zhenghui Wang ${ }^{2}$ \\ Zhuangqun Yang ${ }^{3}$ \\ 'Department of Dermatology, The \\ Second Affiliated Hospital, Xi'an \\ Jiaotong University, Xi'an, People's \\ Republic of China; ${ }^{2}$ Department of \\ Otolaryngology-Head and Neck \\ Surgery, The Second Affiliated \\ Hospital, Xi'an Jiaotong University, \\ Xi'an, People's Republic of China; \\ ${ }^{3}$ Department of Plastic and Burns \\ Surgery, The First Affiliated Hospital, \\ Xi'an Jiaotong University, Xi'an, \\ People's Republic of China
}

\begin{abstract}
Hemangioma is a common benign tumor affecting infants. In this study, we prepared sodium morrhuate immunoliposomes through encapsulation of sodium morrhuate with liposomes coupled with an anti-VEGFR2/KDR antibody and examined its effect on the biology of human hemangioma endothelial cells (HECs). It was found that compared to the liposomal sodium morrhuate group, treatment with sodium morrhuate immunoliposomes facilitated cell detachment and apoptotic death. Confocal microscopy analysis revealed that sodium morrhuate immunoliposomes had a higher binding activity to HECs than liposomal sodium morrhuate. Apoptosis analysis further demonstrated that treatment with liposomal sodium morrhuate or sodium morrhuate immunoliposomes significantly induced apoptosis in HECs, compared to the control group. Western blot analysis revealed an induction of caspase- 3 and caspase- 9 levels and reduction of caspase- 8 and Bcl-2 levels in HECs treated with liposomal sodium morrhuate or sodium morrhuate immunoliposomes. Taken together, these results indicate that sodium morrhuate immunoliposomes have an increased capacity to target HECs and promote mitochondrial apoptosis. Therefore, sodium morrhuate immunoliposomes may represent a promising agent in the treatment of hemangiomas.
\end{abstract}

Keywords: liposome, hemangioma, VEGF2, KDR, apoptosis

\section{Introduction}

Hemangioma is a common benign tumor affecting infants, with an incidence of $3 \%-8 \%$. $^{1}$ Oral and maxillofacial hemangiomas account for $60 \%$ of all hemangiomas. ${ }^{2}$ The life span of hemangiomas consists of proliferating phase, involuting phase, and involuted phase. ${ }^{3,4}$ Although most hemangiomas can involute spontaneously, this process may take a long time and incur residual scars or pigmentary changes. ${ }^{5}$ Deciphering the mechanisms governing hemangioma growth and progression is of significance in treating this disease.

Local administration of sodium morrhuate is an effective therapy for hemangiomas. ${ }^{6,7}$ Sodium morrhuate has the ability to disrupt endothelial cells and promote blood coagulation and thrombosis. ${ }^{8}$ Many reports proved that sodium morrhuate is effective in treating oral and maxillofacial hemangiomas. ${ }^{9,10}$ For large-size hemangiomas, sodium morrhuate treatment can reduce the lesions and thus facilitate subsequent surgery. ${ }^{11}$ However, sodium morrhuate therapy may cause severe complications such as tissue edema and necrosis. ${ }^{8,12}$ Therefore, many efforts have been made to reduce the side effects of sodium morrhuate in the treatment of hemangiomas.

Apoptosis serves as an important mechanism for clearing of undesired cells. Compelling evidence indicates that apoptosis plays a critical role in the involution of
Correspondence: Zhenghui Wang Zhuangqun Yang

Xi'an Jiaotong University, $157 \mathrm{X}$

Wu Road, Xi'an, Shaanxi 710004,

People's Republic of China

Tel +862987679866

Fax +862987678421

Email ehui4298@163.com;

zhuangqunyang@yahoo.com 
hemangiomas. ${ }^{13,14}$ Our previous studies have demonstrated that liposome-encapsulated sodium morrhuate can promote apoptosis of hemangioma endothelial cells (HECs) in vitro. ${ }^{15}$

The VEGF/VEGFR signaling axis plays an important role in the growth of hemangiomas. VEGF is a potent growth factor for endothelial cells. This cytokine can selectively stimulate endothelial cell mitosis, which is dependent on its binding to the specific receptor on endothelial cells, leading to induction of VEGFR tyrosine phosphorylation. Despite the growth-promoting role in endothelial cells, VEGF has little impact on the growth of vascular smooth muscle cells, suggesting the presence of specific receptors on endothelial cell surface.

In this study, we generated sodium morrhuate immunoliposomes via encapsulation of sodium morrhuate with liposomes coupled with an anti-VEGFR2/KDR antibody. The anti-VEGFR2/KDR antibody-mediated targeting to HECs would improve the therapeutic efficacy of sodium morrhuate and reduce damage to normal cells. ${ }^{16}$ The influence of the sodium morrhuate immunoliposome on the biology of human HECs was evaluated.

\section{Materials and methods Materials}

Dithiothreitol solution, Rhodamine PE, 3-[4, 5-dimethyl2-thiazolyl]-2, 5-diphenyl-2H-tetrazolium bromide (MTT), Annexin V-FITC apoptosis detection kit, N-succinimidyl-3(2-pyridyldithio)propionate (SPDP) were purchased from Sigma-Aldrich (St Louis, MO, USA). Anti-human KDR monoclonal antibody, VEGFR2/KDR, was from R\&D System (Minneapolis, MN, USA). Egg yolk phosphatidylcholine was purchased from Northern Lipids Inc. (Burnaby, BC, Canada). Cholesterol was obtained from Shanghai Chemical Reagent Co. Ltd., (Shanghai, People's Republic of China). Sodium morrhuate was from Shanghai Donghai Pharmaceutical Company (Shanghai, People's Republic of China). RPM1640 medium, TRIzol reagent, and reverse transcription polymerase chain reaction (RT-PCR) reagent kit were obtained from Invitrogen (Grand Island, NY, USA). Anti-VIII antibody, biotinylated IgG, pro-caspase-3, procaspase-8, pro-caspase-9, Bcl-2, Bax, and $\beta$-actin were from Santa Cruz Biotechnology Co., Ltd (Dallas, TX, USA).

\section{Preparation of sodium morrhuate immunoliposomes}

The immunoliposomes were composed by following these steps: antibody-thiolated, blowing film, hydration, and filtration. Anti-human-KDR antibody was dissolved in HEPES buffer, which was mixed with SPDP solution $\left(4 \times 10^{-5} \mathrm{mmol} / \mathrm{mL}\right)$. SPDP/Ab ratio was about 15:1 (molar ratio), vibrated in the oscillator, and PDP-Ab was obtained. Then unpaired SPDP was removed through the Amicon Ultra-4 tube. The DTT solution $(0.6483 \mathrm{mmol} / \mathrm{mL})$ was added and made the antibody thiolated. Antibodythiolated solution was slowly added to Mal-PEG-DSPE $\left(8.4994 \times 10^{-5} \mathrm{mmol} / \mathrm{mL}\right)$ in a vacuumed tube to form a homogeneous mixture with vibration, which was named Ab-S-Mal-PEG-DSPE. Sodium morrhuate was dissolved in HEPES buffer, mixed with the antibody-phospholipid solution, and filtered with a $400 \mathrm{~nm}$ strainer two times, a $200 \mathrm{~nm}$ strainer two times and a $100 \mathrm{~nm}$ strainer five times until formation of suspension. The Sephadex G-50 column of ultrafiltration was used to remove unpaired sodium morrhuate. The cysteine solution was added for sealing the unreacted maleimide. Then, the sodium morrhuate immunoliposomes were obtained. The immunoliposomes were stored under nitrogen atmosphere for 3 and 6 months at $4^{\circ} \mathrm{C}$ and the apparent characteristics were observed. The total amount of sodium morrhuate loaded in $0.5 \mathrm{~mL}$ immunoliposomes was determined by high-performance liquid chromatography (HPLC) after extraction with $2 \mathrm{~mL}$ ethanol. The amount of free sodium morrhuate was analyzed by HPLC after suspending $0.5 \mathrm{~mL}$ of immunoliposomes in $2 \mathrm{~mL}$ water. The encapsulation efficiency of sodium morrhuate was calculated from the following equation: $\left(1-\mathrm{C}_{\text {free }} / \mathrm{C}_{\text {total }}\right) \times 100 \%$, where $\mathrm{C}_{\text {free }}$ and $\mathrm{C}_{\text {total }}$ represent the concentrations of free and total sodium morrhuate, respectively.

\section{Culture of HECs}

All procedures were approved by the Ethics Review Board of The Second Affiliated Hospital of Xi' an Jiaotong University (permit number: 2015-17). All the patients signed the informed consent form before surgery. Proliferating phase HECs were obtained from the Department of Pediatric Surgery, The Second Affiliated Hospital, Xi'an Jiaotong University (Xi'an, People's Republic of China). Cells were cultured in RPM1640 medium supplemented with 10\% fetal bovine serum. After reaching confluence, the cells were subcultured. Cells at passages 5 and 6 were used in this study. ${ }^{15}$

\section{RT-PCR analysis}

Total RNA was extracted from HECs using TRIzol reagent and reverse transcribed to first-strand cDNA using the reverse RT-PCR reagent kit. Polymerase chain reaction (PCR) primers used are as follows: VIII gene: forward 5'-CCAACATGATGGCATGGAAG-3' and reverse 5'-CGAGGACTAAGGGAGCATA-3'; VEGFR2: forward 
5'-AACGTGTCACTTTGTGCAAGA-3' and reverse 5'-TTCCATGAGACGGACTCAGAA-3'; $\beta$-actin: forward 5'-GAGGGAAATCGTGCGTGAC-3' and reverse 5'-TAGGAGCCAGGGC AGTAATCT-3'. $\beta$-Actin was used as a loading control. PCR products were separated on $1.5 \%$ agarose gels and visualized by ethidium bromide staining. The images were analyzed by the GEL DOC 2000 system (Bio-Rad, Hercules, CA, USA).

\section{Confocal microscopy}

For confocal microscopy, immunoliposomes were prepared by incorporation of a fluorescent dye Rhodamine PE. Fluorescence-labeled phosphatidylethanolamine (Rho-PE) were added according to the proportion (EPC $100 \mathrm{mg}$, CHO $37.69 \mathrm{mg}$, and Rho-PE $0.3 \mathrm{mg}$ ), which were dissolved $2 \mathrm{~mL}$ chloroform, then nitrogen blowed the chloroform and completely removed the chloroform. Cells were seeded onto coverslips in 6-well plates and at $80 \%$ confluence, added with immunoliposomes or culture medium, and incubated for $2 \mathrm{~h}$ at $4{ }^{\circ} \mathrm{C}$. After washing, the cells were added to fresh culture medium and cultured at $37^{\circ} \mathrm{C}$ for additional $30 \mathrm{~min}$. Cells were then fixed and imaged with a confocal microscope.

\section{Giemsa staining and immunocytochemistry}

For Giemsa staining, when cells were confluent for about $80 \%$ on coverslip, slides were fixed in methanol and Giemsa stain was diluted with deionized water in a ratio of 1:20. The slides were then rinsed in deionized water. The slides were air dried and Giemsa staining was evaluated. Cells were plated on coverslips and allowed to grow to $80 \%$ confluence. Cells were washed and fixed with $4 \%$ paraformaldehyde for $30 \mathrm{~min}$. Normal goat serum was used to block nonspecific binding sites. The coverslips were then incubated at $4^{\circ} \mathrm{C}$ overnight with anti-human VEGFR2/KDR and anti-VIII antibodies, followed by incubation with biotinylated IgG. Bound antibodies were visualized using 3,3'-diaminobenzidine. The coverslips were mounted and examined under a microscope.

\section{In vitro cytotoxic assay}

HECs were seeded onto 96-well plates $\left(1 \times 10^{4}\right.$ cells/well $)$ and treated with different concentrations of immunoliposomes for $24 \mathrm{~h}$. Untreated cells were used as control. The MTT solution $(0.5 \mathrm{mg} / \mathrm{mL})$ was added to each well and incubated for $4 \mathrm{~h}$ at $37^{\circ} \mathrm{C}$. Absorbance at $490 \mathrm{~nm}$ was measured using a microplate reader.

\section{Apoptosis detection by flow cytometry}

HECs were seeded onto 6 -well plates $\left(1 \times 10^{6}\right.$ cells/well $)$ and treated with different concentrations of immunoliposomes for
$24 \mathrm{~h}$. Cell apoptosis was detected by flow cytometry using Annexin V-FITC apoptosis detection kit.

\section{Transmission electron microscopy}

Sodium morrhuate immunoliposome solution was diluted 10 and 100 times. The sample was poured onto a wan copper nets and stained with $2 \%$ phosphotungstic acid. After drying at room temperature, the nets were examined with an electron microscope (TEM-2000EX; JEOL, Tokyo, Japan). For examination of cell morphology, HECs were prefixed with $2.5 \%$ glutaraldehyde in $0.1 \mathrm{M}$ phosphate buffer and postfixed in $1 \%$ osmium tetroxide. The samples were dehydrated in an ascending series of ethanol to $100 \%$, embedded, and cut into ultrathin sections. Sections were stained with $0.5 \%$ uranyl acetate and saturated lead citrate, and examined by transmission electron microscopy.

\section{Western blot analysis}

Cells were treated with sodium morrhuate immunoliposomes for $48 \mathrm{~h}$ and lysed in lysis buffer. After clearing by centrifugation at $12,000 \times g$ at $4^{\circ} \mathrm{C}$ for $3 \mathrm{~min}$, the protein samples were separated by sodium dodecyl sulfate polyacrylamide gel electrophoresis and transferred onto nitrocellulose membranes. The membranes were incubated with the primary antibodies recognizing pro-caspase-3, procaspase-8, pro-caspase-9, Bcl-2, Bax, and $\beta$-actin overnight at $4^{\circ} \mathrm{C}$, followed by horseradish peroxidase-conjugated secondary antibodies. Protein bands were detected with the enhanced chemiluminescent reagent (Millipore, Etobicoke, ON, Canada) and quantified with GEL DOC 2000 System.

\section{Statistical analysis}

All experiences were performed in triplicates. Data are presented as mean $\pm \mathrm{SD}$, and were analyzed by one-way analysis of variance test. The value of $p<0.05$ was considered statistically significant.

\section{Results}

\section{Characterization of sodium morrhuate immunoliposomes}

Figure1A shows the schematic illustration of the liposomes which are labeled with the anti-VEGF2 monoclonal antibody. The immunoliposomes showed a Gaussian distribution in size, with a mean diameter of $122.9 \mathrm{~nm}$ (Figure 1B). A nucleolar and lipid-coating structure was obvious. The drug encapsulation efficiency was $96.34 \%$. When the sodium morrhuate immunoliposomes were diluted 100 times, they showed a typical liposome morphology under a transmission electron microscope (Figure 1C). The immunoliposomes 


\section{A}

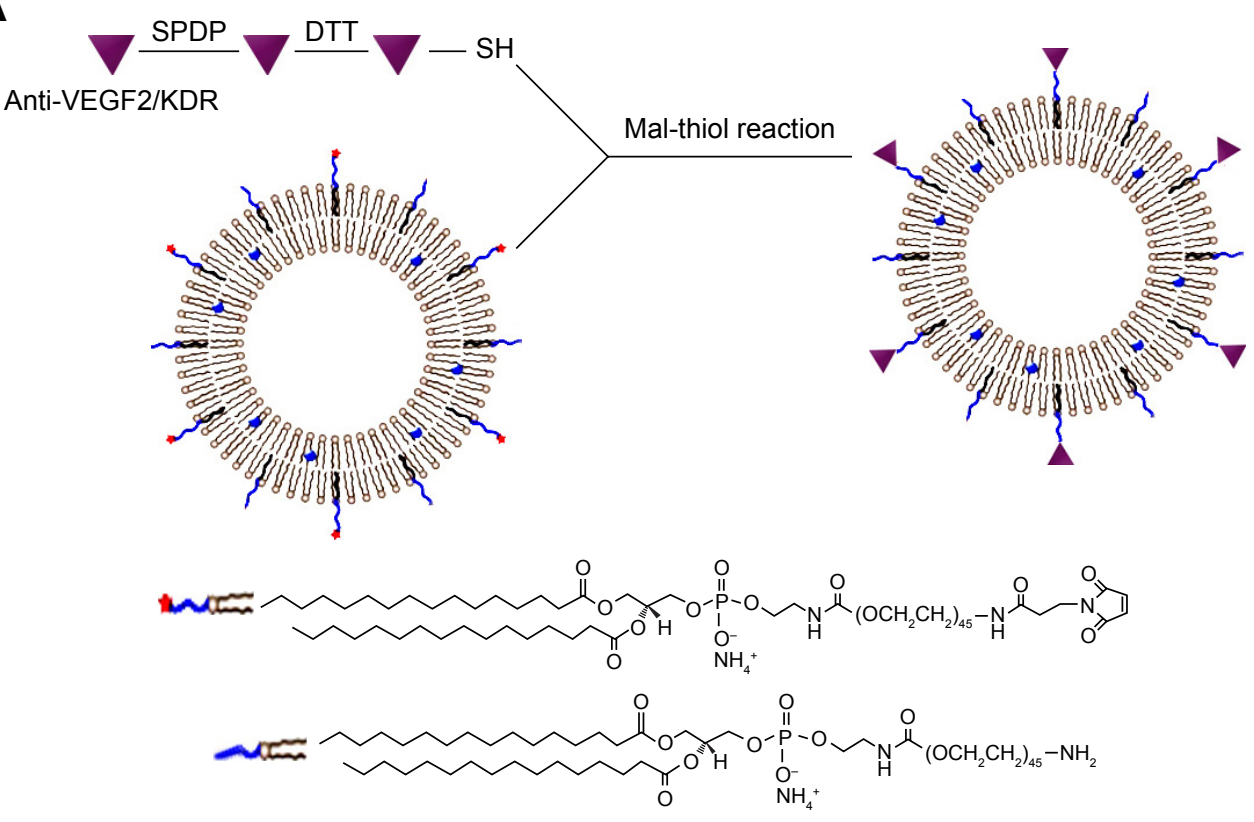

B

C
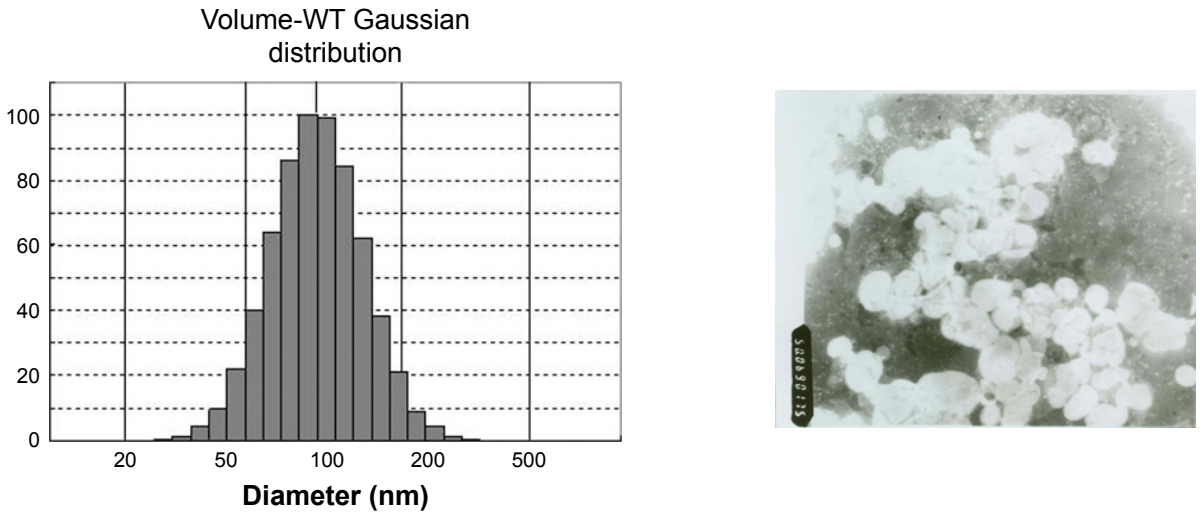

Figure I Preparation of liposomes coupled with anti-VEGF2. (A) Schematic illustration of the coupling of anti-VEGF2 to liposomes via maleimide-thiol reaction. (B) Particle size distribution of liposomes with anti-VEGF2. (C) TEM examination revealed a uniform spherical shape for the liposomes coupled with anti-VEGF2 (magnification $\times 5,000$ ). Abbreviations: Diam, diameter; Mal, maleimide; SPDP, N-succinimidyl-3-(2-pyridyldithio)propionate; TEM, transmission electron microscopy; VEGF2, vascular endothelial growth factor 2; WT, wild type.

remained stable and did not show obvious changes in appearance after a 6-month storage at $4{ }^{\circ} \mathrm{C}$.

\section{Characterization of HECs}

The cell cultures were positive for factor VIII (Figure 2A and B). Reverse transcription PCR analysis demonstrated that the isolated cells expressed factor VIII and VEGF2 mRNA (Figure 2C).

\section{Morphological changes}

Figure 3A showed the control group. Sodium morrhuatetreated HECS showed the formation of vacuoles $80 \mathrm{~min}$ after treatment and underwent necrosis $160 \mathrm{~min}$ later (Figure 3B). While in the blank liposome group, cells retained a normal morphology (Figure 3C). From 120 min after liposomal sodium morrhuate treatment, cells became round and
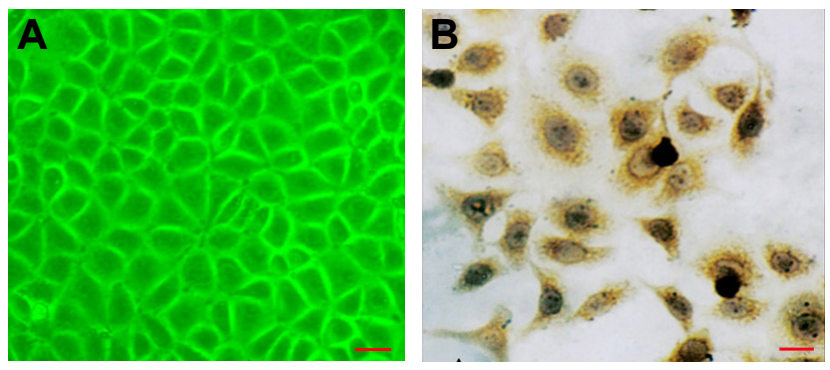

C

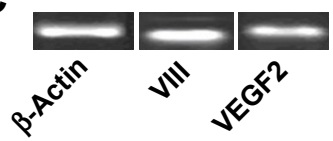

Figure 2 Characterization of HECs. (A) Morphology of HECs. (B) Immunocytochemistry showed that the cell cultures were positive for factor VIII. (C) Reverse transcription PCR analysis demonstrated that the isolated cells expressed factor VIII and VEGF2 mRNA. Bar $=50 \mu \mathrm{m}$.

Abbreviations: HEC, hemangioma endothelial cell; PCR, polymerase chain reaction; VEGF2, vascular endothelial growth factor 2 . 


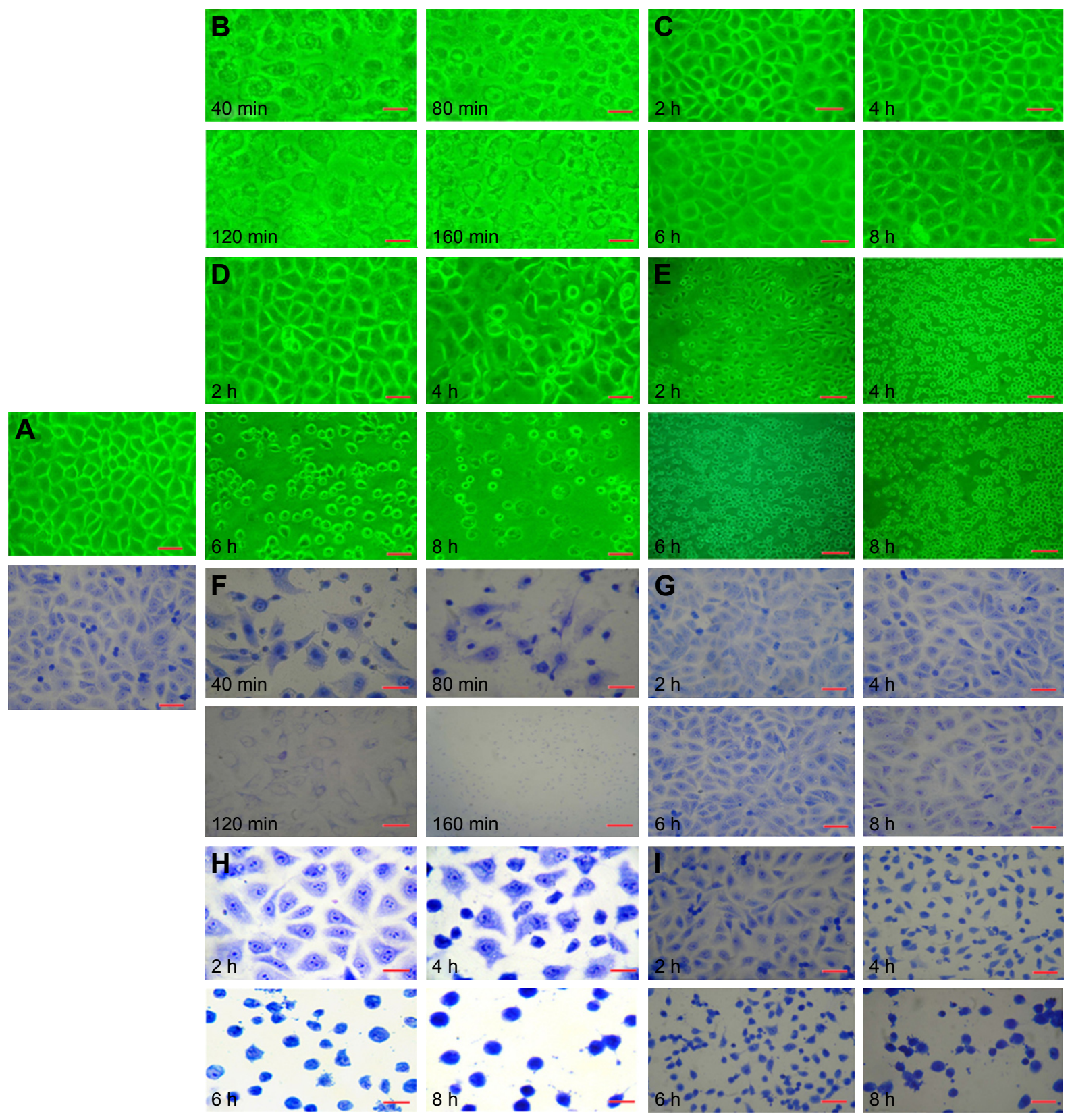

Figure 3 Morphological characteristics of HECs (microscope and Giemsa staining). (A) Control group; (B and F) sodium morrhuate group; (C and G) blank liposome group; $(\mathbf{D}$ and $\mathbf{H}$ ) liposomal sodium morrhuate group; $(\mathbf{E}$ and $\mathbf{I})$ sodium morrhuate immunoliposome group. Scale bars $=50 \mu \mathrm{m}$.

Abbreviation: HEC, hemangioma endothelial cell.

detached, but had an intact plasma membrane (Figure 3D). Compared to the liposomal sodium morrhuate group, treatment with sodium morrhuate immunoliposomes facilitated cell detachment and apoptotic death (Figure 3E). Giemsa staining confirmed the morphological changes in the different groups (Figure 3F-I).

\section{Targeting of sodium morrhuate immunoliposomes to HECs in vitro}

Examination by confocal microscopy revealed that compared to liposomal sodium morrhuate, sodium morrhuate immunoliposomes had an enhanced binding activity to HECs (Figure 4).

\section{Cytotoxic assays}

Compared to the control group, sodium morrhuate treatment led to a marked reduction in the number of viable cells (Figure 5). Similarly, the number of viable cells was reduced after treatment with liposomal sodium morrhuate and at a faster rate in the sodium morrhuate immunoliposome group. Apoptosis analysis further demonstrated that treatment with liposomal sodium morrhuate or sodium morrhuate 

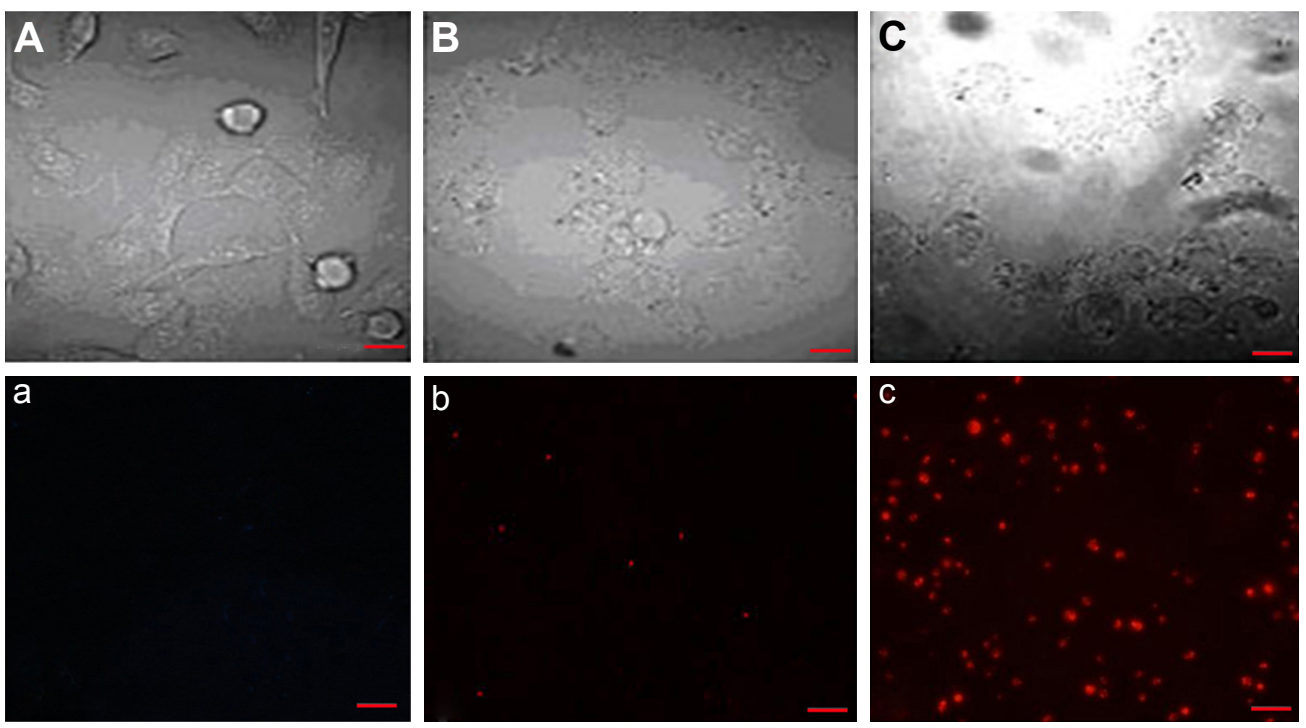

Figure 4 Confocal microscopy reveals the targeting of sodium morrhuate immunoliposomes to HECs. (A and a) Control group; (B and b) liposomal sodium morrhuate group; ( $\mathbf{C}$ and c) sodium morrhuate immunoliposomes group. Scale bars $=50 \mu \mathrm{m}$.

Abbreviation: HEC, hemangioma endothelial cell.

immunoliposomes significantly induced apoptosis in HECs, compared to the control group (Figure 6).

\section{Transmission electron microscopy examination}

Sodium morrhuate-treated HECs displayed swollen organelles and disruption of plasma membrane, indicative of necrotic features (Figure 7A and B). By contrast, HECs treated

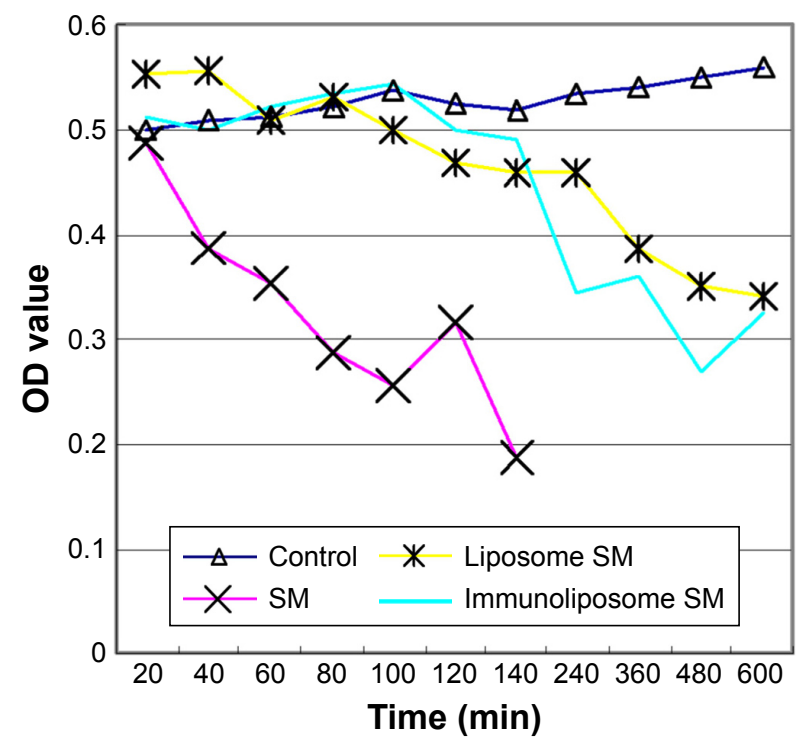

Figure 5 HECs were treated with indicated agents for different times and tested for viability by MTT assay.

Abbreviations: HEC, hemangioma endothelial cell; MTT, 3-[4, 5-dimethyl-2thiazolyl]-2, 5-diphenyl-2H-tetrazolium bromide; OD, optical density; SM, sodium morrhuate. with liposomal sodium morrhuate or sodium morrhuate immunoliposomes exhibited typical apoptotic features, including chromatin condensation and fragmentation and formation of apoptotic bodies (Figure 7C and D) compared with the control group (Figure 7E).

\section{Protein levels detected by western blot analysis}

Western blot analysis revealed that compared to the control group, caspase- 3 and caspase-9 levels were increased and caspase- 8 and Bcl-2 levels were decreased in HECs treated with liposomal sodium morrhuate or sodium morrhuate immunoliposomes (Figure 8). However, the level of Bax did not differ among the groups studied.

\section{Discussion}

Most of hemangiomas are benign and can spontaneously regress over time. However, some may cause residual scarring. ${ }^{17}$ In 1983, Finn et al suggested complete involution of hemangiomas as absence of scarring and capillary dilation. They found that $80 \%$ of the hemangiomas that disappeared after the age of 6 years caused evident abnormalities, whereas only $38 \%$ of those that disappeared by the age of 6 years caused the problem. ${ }^{18}$ Severe complications of hemangiomas include ulceration, bleeding, infection, coagulation disorder, and congestive heart failure. ${ }^{19,20}$ Therefore, early treatment is of significance for problematic hemangiomas.

Several agents such as ethanol, pingyangmycin, urea, and sodium morrhuate have been used to treat hemangiomas. ${ }^{6,21}$ 


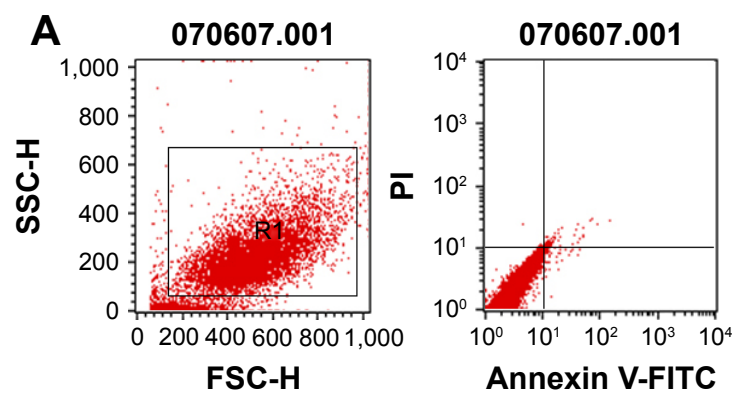

File: 070607.001

\begin{tabular}{|lll|}
\hline Quad & $\%$ gated & $\%$ total \\
\hline UL & 0.21 & 0.17 \\
UR & 1.00 & 0.80 \\
LL & 97.78 & 77.97 \\
LR & 1.00 & 0.80 \\
\hline
\end{tabular}

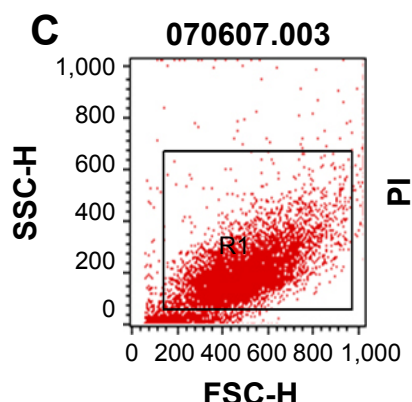

FSC-H

\begin{tabular}{|lll|}
\hline Quad & \% gated & $\%$ total \\
\hline UL & 0.09 & 0.07 \\
UR & 2.94 & 2.22 \\
LL & 66.01 & 49.80 \\
LR & 30.95 & 23.35 \\
\hline
\end{tabular}
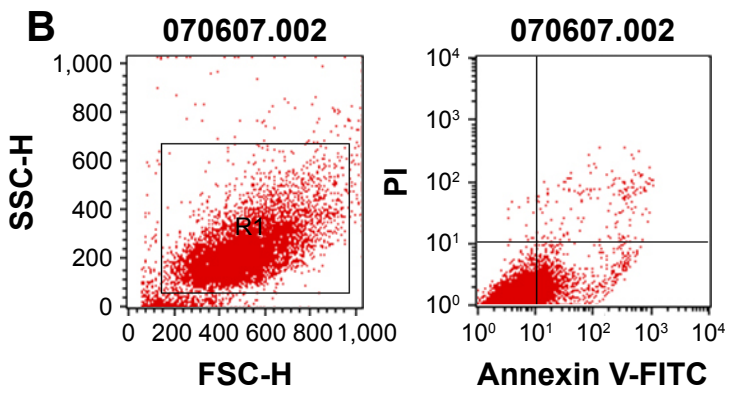

File: 070607.002

\begin{tabular}{|lll|}
\hline Quad & $\%$ gated & $\%$ total \\
\hline UL & 0.16 & 0.14 \\
UR & 1.99 & 1.75 \\
LL & 78.24 & 68.92 \\
LR & 19.62 & 17.28 \\
\hline
\end{tabular}

D

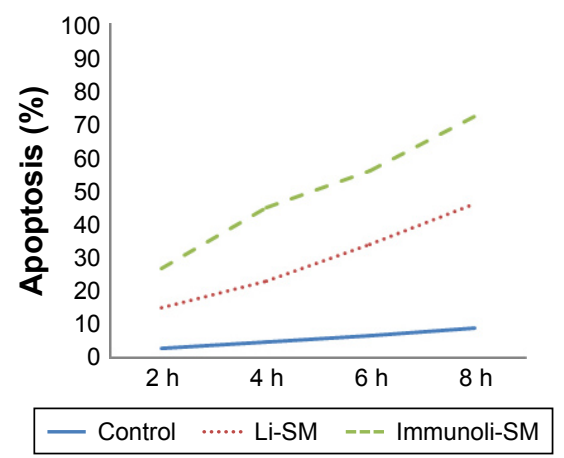

Figure 6 Apoptosis analysis by flow cytometry. (A) Control group; (B) liposomal sodium morrhuate group; (C) sodium morrhuate immunoliposomes group; (D) quantification of apoptosis.

Note: The sample number is show above each analysis.

Abbreviations: Li, liposome; SM, sodium morrhuate.

Despite effective reduction of lesions, sodium morrhuate may cause severe complications such as tissue edema, necrosis, and allergies. Our previous work demonstrated that liposomal sodium morrhuate caused a lower toxicity than sodium morrhuate and preferentially induced apoptotic death. ${ }^{15}$ Liposomal delivery system has several advantages such as enhanced drug solubility, low side effects, prolonged drug release, and reduced drug degradation. ${ }^{22}$ However, the targeting capacity of this delivery system is poor. ${ }^{23}$ The application of immunoliposomes can selectively deliver drugs to target cells and is attracting increasing interest. ${ }^{23,24}$ In the present study, SPDP was used as a cross-linker and a thiolated antiKDR antibody was conjugated to phosphatidylethanolamine. SPDP is effective in adding single active groups. Hansen et al revealed that thiolation can retain antibody activity and facilitate the conjugation to polyethylene glycol. ${ }^{25}$ Pan et al used the same strategy to prepare an immunoliposome to target the epidermal growth factor receptor (EGFR) on glioma cells. ${ }^{26}$ They found that the EGFR-targeting immunoliposomes had a good targeting capacity. The Gaussian distribution in size suggests a high stability for immunoliposomes.

Increasing evidence indicates overexpression of VEGF and its receptors in proliferating hemangiomas. ${ }^{27}$ Many researchers have proved that the VEGF/VEGFR signaling axis plays a critical role in the growth of hemangiomas. ${ }^{27,28}$ To date, three VEGFRs have been identified: VEGFR1 (also known as flt-1), VEGFR2 (also known as flk-1/KDR), and VEGFR3 (also known as flt-4). Kinase insert domain receptor (KDR, a type II receptor tyrosine kinase) is the major receptor involved in VEGF activity and shows a high affinity to $\mathrm{VEGF}{ }^{28}$ It is well accepted that VEGFR2/KDR mediates the inductive effect of VEGF on endothelial cell growth and 

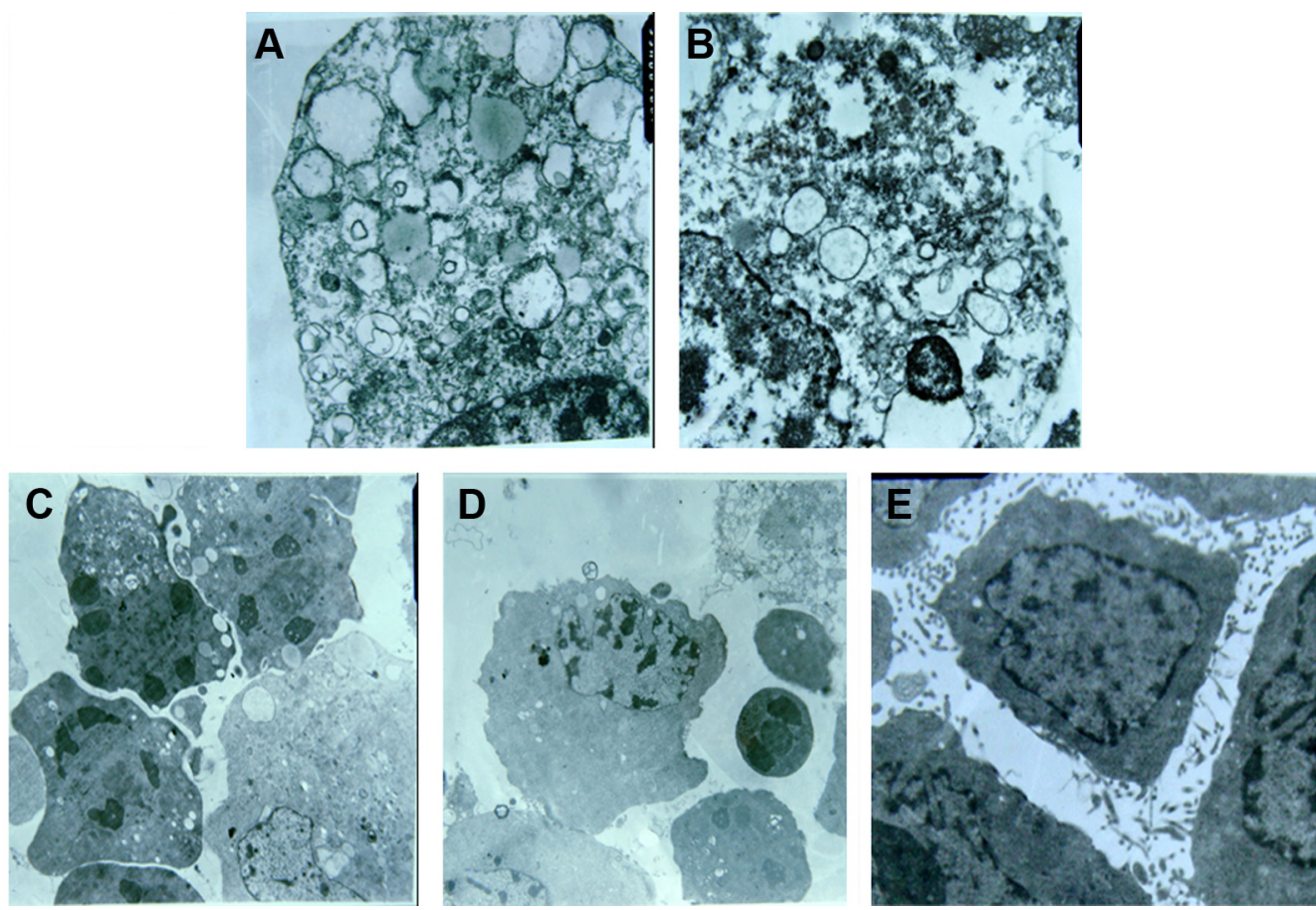

Figure 7 TEM examination of HECs treated with indicated agents. (A and B) Sodium morrhuate group $(\times 10,000)$; $(\mathbf{C})$ liposomal sodium morrhuate group $(\times 4,000)$; (D) sodium morrhuate immunoliposome group $(\times 4,000)$; (E) control group $(\times 4,000)$.

Abbreviations: HEC, hemangioma endothelial cell; TEM, transmission electron microscopy.

differentiation. ${ }^{29}$ After ligation with VEGF, VEGFR2/KDR interacts with $\mathrm{SH} 2$ domain-containing adaptor such as Grb2, consequently leading to the activation of MAPK and downstream growth-related genes. In this study, we showed that the VEGFR2-targeting immunoliposomes had a high binding activity to endothelial cells.

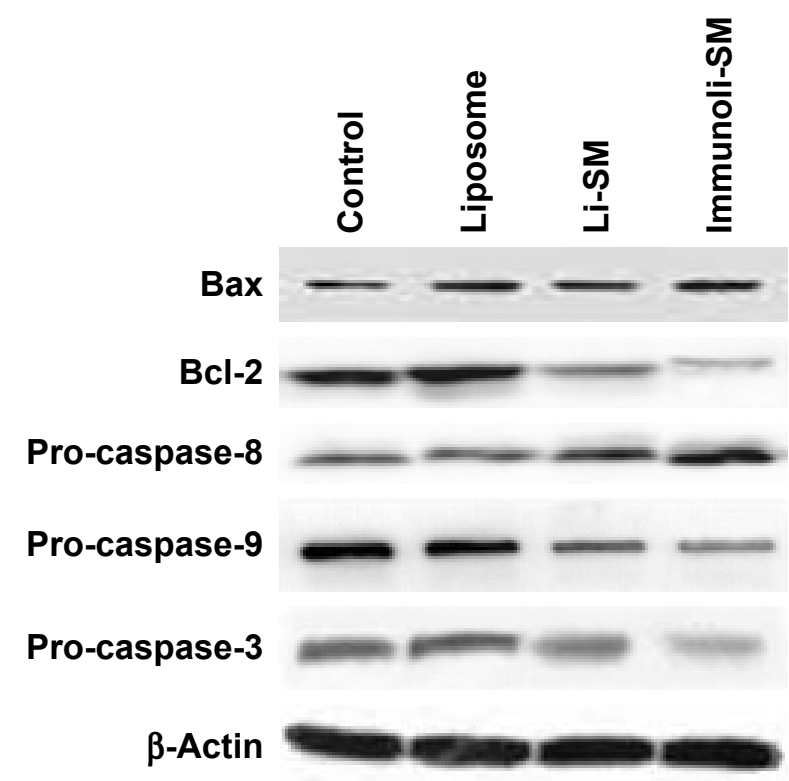

Figure 8 Western blot analysis of indicated proteins from different groups. Representative blots of three independent experiments with similar results are shown. Abbreviations: Li, liposome; SM, sodium morrhuate.
Endothelial cell apoptosis is an important mechanism for the involution of hemangiomas. ${ }^{30}$ Apoptosis is a complex biological process that is coordinated by many genes and signaling pathways. It has been reported that multiple genes such as Bcl-2 family members, p53, Fas, c-myc, and k-ras are involved in the regulation of apoptosis. There are two major apoptotic pathways: extrinsic death receptor and intrinsic mitochondrial pathways. ${ }^{31}$ Both pathways lead to apoptotic death through activation of a family of cysteine proteases, named caspases, especially caspase- 3 , an effector caspase. In this study, we showed that treatment with liposomal sodium morrhuate or sodium morrhuate immunoliposomes caused an increase of caspase- 3 and caspase- 9 levels and decrease of caspase- 8 levels in HECs. The Bcl-2 family members (eg, anti-apoptotic proteins Bcl-2 and Bcl-xL and pro-apoptotic proteins $\mathrm{Bax}$ and $\mathrm{Bcl}-\mathrm{xs}$ ) contribute to mitochondrial apoptosis. We found that the Bcl-2 levels were decreased after treatment with liposomal sodium morrhuate or sodium morrhuate immunoliposomes, but Bax remained unchanged. These findings indicate that sodium morrhuate immunoliposomes induce mitochondrial apoptosis in HECs, which involves the regulation of Bcl-2 family members.

\section{Conclusion}

KDR-targeting sodium morrhuate immunoliposomes have an increased capacity for binding to HECs compared to 
liposomal sodium morrhuate and can elicit apoptotic death through the mitochondrial apoptotic cascade.

\section{Acknowledgments}

We thank for Dr Xiaoyan Zhao for her technical assistance. This study was supported by the Fundamental Research Funds for the Central Universities, the Funds for the Second Hospital of Xi' an Jiaotong University (YJ (ZD) 201103), and the Funds for Shaanxi Province Ke Ji Gong Guan (2016SF-073, 2017SF-161).

\section{Disclosure}

The authors report no conflicts of interest in this work.

\section{References}

1. Smolinski KN, Yan AC. Hemangiomas of infancy: clinical and biological characteristics. Clin Pediatr (Phila). 2005;44(9):747-766.

2. Buckmiller LM, Richter GT, Suen JY. Diagnosis and management of hemangiomas and vascular malformations of the head and neck. Oral Dis. 2010;16(5):405-418.

3. Przewratil P, Sitkiewicz A, Wyka K, Andrzejewska E. Serum levels of vascular endothelial growth factor and basic fibroblastic growth factor in children with hemangiomas and vascular malformations - preliminary report. Pediatr Dermatol. 2009;26(4):399-404.

4. Mulliken JB, Glowacki J. Hemangiomas and vascular malformations in infants and children: a classification based on endothelial characteristics. Plast Reconstr Surg. 1982;69(3):412-422.

5. Hemangioma Investigator Group, Haggstrom AN, Drolet BA, Baselga E, et al. Prospective study of infantile hemangiomas: demographic, prenatal, and perinatal characteristics. J Pediatr. 2007;150(3):291-294.

6. He W, Gu AL, Shang JL. [Comparison of the clinical therapeutic effects between local injection of pingyangmycin and sodium morrhuate on oral and maxillofacial venous malformation]. Zhonghua Kou Qiang $Y i$ Xиe Za Zhi. 2007;42(5):308-309. Chinese.

7. Shou BQ, Shou WD, Meng ZY, Yang Z, Zhang SL. [The effect of pinyangmycin, dexamethasonum and sodium morrhuate injected concomitantly to treat cavernous hemangioma]. Shanghai Kou Qiang Yi Xue. 2003;12(1):9-11. Chinese.

8. Shou BQ, Yang Z, Meng ZY, Huang DB, Zheng H, Ding GQ. [Clinical summary of relatively large dose of sodium morrhuate injection for the treatment of maxillofacial cavernous hemangioma: a report of 764 cases]. Shanghai Kou Qiang Yi Xue. 1996;5(2):71-73. Chinese.

9. Zhou K, Liang C, Yang K, Wang L. [A randomized controlled study on the efficacy of modified sclerotherapy in treating angioma of ear, nose and throat]. Lin Chuang Er Bi Yan Hou Ke Za Zhi. 2002;16(12): 681-683. Chinese.

10. Morgan JF, Schow CE Jr. Use of sodium morrhuate in the management of hemangiomas. J Oral Surg. 1974;32(5):363-366.

11. Guo J, Wu H. [Changes of cultured human vein endothelial cells (HVEC) in the presence of therapeutic agents of cavernous hemangioma]. Hua Xi Kou Qiang Yi Xue Za Zhi. 2000;18(5):310-313. Chinese.

12. Fu J, Liu Z, Chen X. Acral necrosis induced by sodium morrhuate sclerotherapy in infantile haemangioma: a case report. J Hand Surg Eur Vol. 2017;42(2):206-207.

13. He X, Liu Y, Li K, Yang A, Wang R, Liu S. Sildenafil suppresses the proliferation and enhances the apoptosis of hemangioma endothelial cells. Exp Ther Med. 2017;13(6):2645-2650.
14. Wnęk A, Andrzejewska E, Kobos J, Taran K, Przewratil P. Molecular and immunohistochemical expression of apoptotic proteins Bax, Bcl-2 and Caspase 3 in infantile hemangioma tissues as an effect of propranolol treatment. Immunol Lett. 2017;185:27-31.

15. Tu JB, Wang ZH, Song Y, et al. [The effect of lipo-sodium morrhuate on human hemangioma endothelial cells]. China J Oral Maxillofac Surg. 2007;5(6):445-450. Chinese.

16. Takahashi K, Mulliken JB, Kozakewich HP, Rogers RA, Folkman J, Ezekowitz RA. Cellular markers that distinguish the phases of hemangioma during infancy and childhood. J Chin Invest. 1994;93(6): 2357-2364.

17. Darrow DH, Greene AK, Mancini AJ, Nooper AJ. Diagnosis and management of infantile hemangioma. Pediatrics. 2015;136(4): e1060-e1104.

18. Finn MC, Glowacki J, Mulliken JB. Congenital vascular lesions: clinical application of a new classification. J Pediatr Surg. 1983;18(6): 894-900.

19. Rangwala S, Wysong A, Tollefson MM, Khuu P, Benjamin LT, Bruckner AL. Rapidly involuting congenital hemangioma associated with profound, transient thrombocytopenia. Pediatr Dermatol. 2014; 31(3):402-404.

20. Lyons LL, North PE, Mac-Moune Lai F, Stoler MH, Folpe AL, Weiss SW. Kaposiform hemangioendothelioma: a study of 33 cases emphasizing its pathologic, immunophenotypic, and biologic uniqueness from juvenile hemangioma. Am J Surg Pathol. 2004;28(5):559-568.

21. Zhang W, Zhang X, Ren X, et al. [Effects of intervention embolization therapy and intratumor injection with hardener on head and neck gigantic hemangioma]. Lin Chuang Er Bi Yan Hou Ke Za Zhi. 2005; 19(3):117-118. Chinese.

22. Wang Z, Li J, Xu X, Duan X, Cao G. Urea immunoliposome inhibits human vascular endothelial cell proliferation for hemangioma treatment. World J Surg Oncol. 2013;11:300.

23. Li Y, Yuan J, Yang Q, et al. Immunoliposome co-delivery of bufalin and anti-CD40 antibody adjuvant induces synergetic therapeutic efficacy against melanoma. Int J Nanomedicine. 2014;9:5683-5700.

24. Sharma G, Anabousi S, Ehrhardt C, Ravi Kumar MN. Liposomes as targeted drug delivery systems in the treatment of breast cancer. J Drug Target. 2006;14(5):301-310.

25. Hansen CB, Kao GY, Moase EH, Zalipsky S, Allen TM. Attachment of antibodies to sterically stabilized liposomes: evaluation, comparison and optimization of coupling procedures. Biochim Biophys Acta. 1995 1239(2):133-144.

26. Pan X, Wu G, Yang W, Barth RF, Tjarks W, Lee RJ. Synthesis of cetuximab-immunoliposomes via a cholesterol-based membrane anchor for targeting of EGFR. Bioconjug Chem. 2007;18(1):101-108.

27. Fu Y, Yang ZG, Zhao LY. Angiogenesis characteristics of infantile hemangioma and feasibility observation of transplantation model of human hemangioma on mice. Eur Rev Med Pharmacol Sci. 2017;21(6): $1276-1280$.

28. Neufeld G, Cohen T, Gengrinovitch S, Poltorak Z. Vascular endothelial growth factor (VEGF) and its receptors. FASEB J. 1999;13(1):9-22.

29. Jopling HM, Odell AF, Pellet-Many C, et al. Endosome-to-plasma membrane recycling of VEGFR2 receptor tyrosine kinase regulates endothelial function and blood vessel formation. Cells. 2014;3(2):363-385

30. Qiu MK, Wang SQ, Pan C, et al. ROCK inhibition as a potential therapeutic target involved in apoptosis in hemangioma. Oncol Rep. 2017; 37(5):2987-2993.

31. Elmore S. Apoptosis: a review of programmed cell death. Toxicol Pathol. 2007;35(4):495-516. 


\section{Publish your work in this journal}

The International Journal of Nanomedicine is an international, peerreviewed journal focusing on the application of nanotechnology in diagnostics, therapeutics, and drug delivery systems throughout the biomedical field. This journal is indexed on PubMed Central, MedLine, CAS, SciSearch $\AA$, Current Contents $\AA /$ Clinical Medicine,

Journal Citation Reports/Science Edition, EMBase, Scopus and the Elsevier Bibliographic databases. The manuscript management system is completely online and includes a very quick and fair peer-review system, which is all easy to use. Visit http://www.dovepress.com/ testimonials.php to read real quotes from published authors.

Submit your manuscript here: http://www.dovepress.com/international-journal-of-nanomedicine-journal 